\title{
HISTORIA REPRODUCTORA Y FECUNDIDAD DE LAS MUJERES CHILENAS DE TIERRA DEL FUEGO.
}

\author{
J OEL PASCUAL，CLARA GARCíA-MORO, MIGUEL HERNÁNDEZ, MARTA MUÑOZ-TUDURí,*
}

\section{RESUMEN}

El presente estudio analiza la información obtenida a través de 182 encuestas de fecundidad a mujeres en edad posreproductora de la Tierra del Fuego chilena. La muestra es representativa de la población femenina fueguina desde casi el inicio de la colonización. Se describen los distintos eventos e intervalos que definen la historia reproductora de estas mujeres, además de sus valores de fecundidad y de fecundidad marital. Su vida reproductiva, toda ella transcurrida en la isla, se caracteriza por un largo intervalo fértil, un corto intervalo fecundo y una reducida fecundidad, y se constata cómo la mayor eficiencia de uso de sus años reproductores se traduce en un tamaño de la progenie mayor. Finalmente, se realizan dos aproximaciones distintas al estudio de los determinantes de la fecundidad de las mujeres de la población: por un lado, el análisis mediante el modelo agregativo de la fecundidad descrito por Bongaarts y Potter $y$, por otro, la aplicación del path analysis. Los patrones de nupcialidad, el ritmo de concepción y las prácticas de anticoncepción son propuestos como los principales determinantes de la fecundidad en las mujeres chilenas de Tierra del Fuego.

PALABRAS CLAVES: Fecundidad, determinantes próximos, path análisis.

\section{WOMEN FERTILITY AND REPRODUCTIVE YEARS IN CHILEAN TIERRA DEL FUEGO}

\section{ABSTRACT}

The present study analyzes 182 interviews to postmenopausal women of the Chilean part of Tierra del Fuego. These women can be considered a representative sample of the population of the region from the beginning of the recent settlement. Events and intervals that explain reproductive lifes of these women are described, and fertility and marital fertility rates are calculated. The reproductive history of these women is characterized by a long fertile span, a short childbearing period, and low fertility. It was observed that the higher the efficiency of use of their reproductive years, the larger the number of surviving children. Finally, two different pathways were chosen to study fertility determinants of these women: Bongaarts' fertility aggregative model and path analysis. Marriage patterns, the rhythm of conception and practices of contraception are proposed as the main determinants of fertility in Tierra del Fuego.

KEYWORDS: Fertility, proximate factors, path analysis.

* Unidad de Antropología, Facultad de Biología, Universidad de Barcelona. Avda. Diagonal 645. 08028 Barcelona. España Email: cgarcia@ub.edu; joelpascual@gmail.com; mhernandez@ub.edu; mmunoz@ub.edu 


\section{INTRODUCCIÓN}

En la dinámica de las poblaciones humanas el balance entre natalidad y mortalidad determina de manera directa la intensidad del crecimiento natural de las poblaciones; además de modificar la estructura por edad de las mismas, hecho que, de manera indirecta, puede condicionar también su crecimiento. El papel clave que la fecundidad tiene en la dinámica de las poblaciones humanas se acrecienta al comprobar que este fenómeno es regulable por los individuos de una población. Aunque los dos miembros de la pareja están ciertamente implicados en la intensidad y regulación de la fecundidad, su estudio suele referirse al análisis de la fecundidad femenina (más que a la fecundidad masculina o de la pareja) debido a que los principales sucesos limitantes en la reproducción se relacionan con la vida reproductora de la mujer (Wood, 1994).

Desde la ecología humana, la regulación de la fecundidad se interpreta como un intento de adaptación biológica y cultural del comportamiento reproductor de una población a las condiciones ambientales, socioeconómicas y culturales que rigen en el medio en el que se desarrolla dicha población. De esta manera, el análisis de los valores y patrones que caracterizan la fecundidad de las poblaciones humanas permite valorar la adaptación de estas sociedades a su entorno.

En antropología biológica han merecido especial atención los estudios de poblaciones pequeñas y con aislamiento geográfico o cultural, ya que en ellas las fuerzas evolutivas operantes son muy similares a las que debieron actuar durante una parte importante de la historia evolutiva de la humanidad. Sin embargo, es notoria la ausencia de modelos que describan específicamente la formación de poblaciones resultantes de migraciones colonizadoras, como es la población chilena de la Isla Grande de Tierra del Fuego. Esta población presenta también algunas particularidades que acrecientan el interés de su estudio. Su carácter insular dota a la población de cierto aislamiento y condiciona la percepción del día a día de sus habitantes. Por su parte, la elevada latitud del territorio y las corrientes marinas y atmosféricas establecen condiciones ambientales extremas para la zona, lo que ha podido determinar en la población ciertas adaptaciones socioculturales. Además, la población actual es el resultado de consecutivas migraciones euro- peas y americanas, que ha derivado en una interesante mezcla biológica y cultural. Así, la caracterización biodemográfica de la población colonizadora de Tierra del Fuego representa una aportación poco frecuente al conocimiento del poblamiento reciente de América. Su interés aumenta al disponer de abundante información demográfica desde la fundación de la población a finales del siglo XIX hasta la actualidad.

Este trabajo se centra en la Tierra del Fuego chilena y sus resultados no son extrapolables al territorio argentino, puesto que la evolución histórica y biodemográfica de la población fueguina argentina es distinta. La provincia chilena de Tierra del Fuego está situada en el extremo sur del continente americano (52.50-55익 latitud sur) y se extiende sobre un total de 18.772 $\mathrm{km}^{2}$ de la isla grande de Tierra del Fuego. La provincia está dividida en tres comunas administrativas: Porvenir (3.321.6 km²), cuya capital es la ciudad del mismo nombre; Primavera $\left(3.956 \mathrm{~km}^{2}\right)$ con sede en la población de Cerro Sombrero y Timaukel (11.494.4 $\mathrm{km}^{2}$ ) con sede en Cameron. En conjunto, la población de Tierra del Fuego es el resultado de diversos contingentes migratorios que se suceden en el tiempo: un primer contingente mayoritariamente europeo (compuesto por inmigrantes llegados desde finales del siglo XIX hasta la década de los años 30 del siglo XX) y otro posterior (con inmigrantes que llegan a la provincia desde la década de los años 30 hasta la de los 70) de predominio de individuos procedentes del archipiélago de Chiloé (Martinic, 1992; Hernández et al., 1998; Martinic, 1999).

El proceso colonizador del territorio fueguino ha venido marcado por tres actividades principales: la búsqueda de oro, la explotación de la ganadería ovina y la explotación petrolífera. En este sentido, el inicio de la colonización de Tierra del Fuego puede fecharse en 1881, con la llegada de grupos compuestos por decenas de mineros aventureros. Paralelamente, entre 1883 y 1896 se destinaron a la explotación pecuaria todos los campos fueguinos aptos para la crianza lanar, alquilándose los derechos de explotación a cuatro grandes sociedades, al contrario de lo sucedido en el territorio continental magallánico en donde la colonización había asumido la forma individual. En 1894 se funda el pueblo de Porvenir, el primer y único poblado que se conocería en el territorio fueguino en más de medio siglo, comenzando con el mismo el poblamiento propiamente urbano de Tierra del Fuego (Martinic, 
1982)

Hacia 1910, se podían distinguir en la población del territorio chileno fueguino dos componentes bien diferenciados: uno, rural, con una importante presencia extranjera (mayoritariamente británica); y otro urbano, de carácter multiétnico, aunque con una marcada presencia croata. La cantidad de croatas llegados conformó sin duda un hecho significativo en la colonización de Tierra del Fuego y, en general, del conjunto de la Región Magallánica, tanto por su número (representan un tercio del total del contingente migratorio establecido en la región) como por su trascendencia en el desarrollo económico y en la formación de la sociedad fueguina. El lapso temporal entre 1920 y 1945 fue el período histórico dorado de la Tierra del Fuego chilena desde el punto de vista social y económico (Martinic, 1982).

El descubrimiento de petróleo en el territorio chileno de Tierra del Fuego el año 1945 marcó un punto de inflexión en el desarrollo de la provincia, principalmente porque supuso la creación de nuevos centros de población en el norte del territorio insular (siendo el principal el campamento de Cerro Sombrero en la Comuna de Primavera) y así contribuyó a la reducción de la atracción focal que ejercía Porvenir hasta entonces en la isla. No obstante, los pobladores de las zonas petrolíferas, proced entes del exterior de Tierra del Fuego en su mayoría, constituyen un tipo de sociedad distinto al de la capital fueguina, cuya población es el centro del presente estudio. Simultáneamente, otro de los elementos a considerar en la decadencia en la que entró Porvenir durante un par de décadas fue el paulatino desarraigo de antiguas familias porvenireñas que pasaron a establecerse en Punta Arenas, la capital regional y ciudad continental, o en el territorio argentino insular más próspero económicamente. La búsqueda de un mejor nivel de vida o la atracción que en forma permanente ejercía la dinámica ciudad de Punta Arenas motivó un fenómeno de alejamiento circunstancial o definitivo del territorio fueguino.

Al entrar en el perío do que transcurre desde 1966 hasta la actualidad, la vida y economía insulares fueron cada vez más dependientes de Punta Arenas debido al centralismo en la conducción de las actividades de la Empresa Nacional del Petróleo y por la autonomía con que se manejaban los empresarios ganaderos establecidos en la capital regional. Al mismo tiempo que se reducía el desarrollo poblacional de las zonas rurales de la isla, Porvenir empezó a resurgir, gracias a medidas y acciones de modernización impulsadas desde el gobierno regional. El censo nacional de 2002 registra su mayor población histórica (4.734 habitantes) -excepción hecha de la transitoria y anormal de carácter militar de 1982-, aunque con niveles de crecimiento modestos, sensiblemente inferiores a los de otros centros urbanos regionales.

\section{MATERIAL Y MÉTODOS}

Este trabajo se basa principalmente en la información obtenida a través de encuestas personales de fecundidad, realizadas durante los meses de septiembre y octubre de 2000, a 182 mujeres en edad posreproductora que vivían mayoritariamente en el núcleo urbano de Porvenir. Las mujeres entrevistadas debían cumplir dos condiciones previas: (1) tener más de 45 años en el momento de la encuesta y (2) haber nacido en la provincia fueguina o haber llegado a ella en su infancia 0 adolescencia. Con la primera condición se reducía potencialmente las mujeres a encuestar, ya que el interés del estudio se ha centrado en mujeres que hubieran finalizado su período reproductor. La muestra final obtenida incluye mujeres nacidas entre 1912 y 1956 con lo que, además, queda representada la población casi desde el inicio del poblamiento. Con la segunda condición se pretendía concentrar el estudio en mujeres que hubieran experimentado su vida reproductora en el territorio fueguino. Las mujeres fueron seleccionadas al azar y todos los barrios y clases sociales de la ciudad están representados. Se accedió a ellas a través de las administraciones locales y de las múltiples asociaciones, centros de madre y el hospital existentes en la ciudad. A pesar de que la muestra analizada pertenece sólo a mujeres que han completado su período reproductor y permanecen vivas y en la población (quedan excluidas, por tanto, las más jóvenes, las que emigran y las que fallecen antes de finalizar su período reproductor), este grupo femenino es una muestra representativa de las mujeres chilenas de Tierra del Fuego con las citadas características.

El diseño de la encuesta responde a la voluntad de obtener la información indispensable general y particular para la caracterización de la vida reproductora de las mujeres del lugar. A grandes rasgos, la encuesta comprende cuatro blo- 
ques en los que se recoge respectivamente información de la mujer, datos de sus uniones conyugales estables, características biológicas de su vida e información de la descendencia alcanzada (para una información más detallada de la metodología empleada y del tratamiento de los datos consultar Pascual 2004).

Bloque 1. Datos de la mujer. El primer bloque incluye información general de la mujer y del origen de sus padres y abuelos. Entre las distintas variables consideradas se incluyen la fecha de nacimiento de la mujer, su origen familiar y las circunstancias de llegada de la mujer a la isla (en caso de no haber nacido en la misma).

Bloque 2. Datos de las uniones. El segundo bloque pretende obtener información acerca de las uniones matrimoniales, consensuales (y esporádicas, si ha habido en su contexto de descendencia) que la mujer haya podido establecer. Se recoge información acerca del inicio (y final, y causa del mismo, si procede) y de la localidad donde se estableció la relación.

Bloque 3. Datos biológicos de la mujer. EI tercer bloque incluye datos biológicos básicos para establecer las características principales de la vida reproductora de la mujer. Por un lado, comprende la edad de menarquia y de menopausia, características que definen la longitud del período teórico fecundo de la mujer. Se obtiene también información acerca de las circunstancias de alcance de la menopausia, especialmente las incidencias de la menopausia natural y quirúrgica, lo que responde a un interés de delimitar con el mayor detalle posible el intervalo fecundo de la mujer. Se obtienen otros datos biológicos de interés como la descripción de abortos espontáneos y los recién nacidos muertos y vivos del conjunto de embarazos de una mujer, así como la longitud de una serie de intervalos de la vida reproductora de la mujer, todos indicadores de interés biológico.

B loque 4. Datos de la progenie. Este último bloque pretende recoger la máxima información acerca de la progenie de la mujer. Entre otros, recoge el orden de paridad de la descendencia, si se dio en circunstancia de embarazo sencillo o gemelar, el sexo, la fecha completa del nacimiento, el lugar de nacimiento y algunos datos sobre el ciclo vital de la progenie.

Estas entrevistas personales han permitido reconstruir la historia reproductora de las mujeres encuestadas y obtener una visión detallada de los patrones de reproducción de la po- blación chilena de Tierra del Fuego.

El análisis estadístico de los diferentes parámetros incluidos en este trabajo se ha realizado mayoritariamente con el paquete estadístico SPSS 10.0 y la hoja de cálculo EXCEL XP. Se han utilizado también las aplicaciones Matemathica, para resolución de sistemas de ecuaciones complejos, Statgraphics, para el análisis de diseños $2^{k}$ y Microstat, para valorar la significación de tests. Para evitar una extensión mayor del presente trabajo, en algunas ocasiones se omiten algunos resultados estadísticos 0 parte de los mismos. Todos los análisis estadísticos requeridos se pueden consultar en detalle en Pascual (2004).

\section{RESULTADOS Y DISCUSIÓN}

\section{A. Historia reproductora.}

L a Figura 1 resume los valores medios para los diversos eventos e intervalos de la historia reproductora de las mujeres fueguinas. Por un lado, la aparición de la primera menstruación (menarquia) y el cese definitivo de los ciclos menstruales (menopausia) se dan a unas edades promedio intermedias entre las descritas en poblaciones humanas (13.85 años para la edad media de menarquia y 48.38 años para la edad media de menopausia) y definen un intervalo fértil medio teórico de 34.12 años, un valor elevado entre los descritos en la bibliografía. No obstante, la esterilización como método anticonceptivo definitivo elegido por un sector de la población femenina adelanta la pérdida de su fertilidad, reduciendo su intervalo fértil por término medio en un $43 \%$. De esta manera, la naturaleza del cese de la fertilidad de las mujeres determina la longitud del su intervalo fértil.

Por otro lado, un suceso determinado en mayor medida por factores socioculturales como es la edad nupcial (en esta población, considerada como la edad de inicio de la unión estable, sea formal o consensual) determina la longitud de diversos eventos e intervalos de la vida reproductora de las mujeres, especialmente de la edad de primera maternidad, y de los intervalos previos a ésta. Así, la relativamente elevada edad nupcial (23.03 años) determina las elevadas longitudes del intervalo temporal que transcurre entre la menarquia y el matrimonio (9.01 años) y del intervalo ginecológico (periodo transcurrido entre la aparición de la primera menstruación y 
el nacimiento del primer hijo), que está íntimamente relacionado con el anterior. El valor del intervalo ginecológico medio, 9.92 años, muestra la importancia del tiempo «perdido»; es decir, no utilizado para tener descendencia en la fase inicial de la vida reproductora. En un sentido similar, el intervalo que transcurre entre la última maternidad y la menopausia es largo entre las mujeres encuestadas (15.85 años) lo que permite también valorar el tiempo «perdido» para tener descendencia al final de la vida reproductora de las mujeres. Así, se contabiliza por término medio que las mujeres fueguinas dejan de destinar a tener descendencia unos 25 años de sus potenciales años fértiles.

La elevada edad nupcial (condicionada por la estructura de la población) también determina la relativamente tardía edad media de primera maternidad entre las mujeres encuestadas (23.66 años). En contraposición, la edad media materna al nacimiento del último hijo es especialmente temprana (32.11 años), lo cual constituye un indicador del control voluntario de la fecundidad en un sector importante de las mujeres de la población. No solamente la esterilización o la menopausia quirúrgica adelantan la edad de última maternidad, sino que existe un porcentaje elevado de mujeres con menopausia natural que muestra una edad temprana de última maternidad. En algunos casos, la separación conyugal por ruptura matrimonial o viudedad puede explicar esta edad temprana aunque, en general, los métodos de anticoncepción eficaces deben tener un papel importante. Además, se constata la ausencia de relación entre la disponibilidad de años fértiles y la edad a la que las mujeres tienen su último hijo. Solamente la edad nupcial tardía retrasa la edad de última maternidad.

Finalmente, el intervalo protogenésico (tiempo medio que transcurre desde el enlace nupcial hasta el primer nacimiento) de 1.95 años muestra que el conjunto de mujeres encuestadas de Tierra del Fuego es el resultado de una fusión de diversos comportamientos a este respecto. Así, por un lado, el $69 \%$ de las mujeres se incorpora rápidamente a la reproducción, en un período inferior a los 20 meses, asimilables a un comportamiento propio de poblaciones de fecundidad natural sin control de natalidad; mientras que por otro se observan indicios del efecto de la edad en la fecundabilidad de las mujeres y de un control de la fecundidad ya en los primeros compases de la vida conyugal, que da lugar a intervalos protogenésicos más largos.

A su vez, las edades de primera y última maternidad definen la longitud del intervalo fecundo, que se muestra corto (8.89 años). De esta manera, y tal como se ha apuntado anteriormente, las mujeres de Tierra del Fuego, a pesar de disfrutar de un intervalo fértil largo, destinan un relativo breve perío do de tiempo (sólo el $26.05 \%$ por término medio) a tener su descendencia. $\mathrm{La}$ longitud de este intervalo depende principalmente del tamaño de la progenie alcanzado y de la longitud de los intervalos intergenésicos (espaciamiento entre los diversos hijos), lo que sugiere una reducida fecundidad de las mujeres de Tierra del Fuego.

\section{B. Valores de fecundidad.}

El resultado de la historia reproductora, definida por los distintos eventos e intervalos comentados en el apartado anterior, es un tamaño medio de la progenie de 3.32 hijos nacidos vivos por mujer (D.E. 1.91, $n=168$ ), siendo 3 el nú-

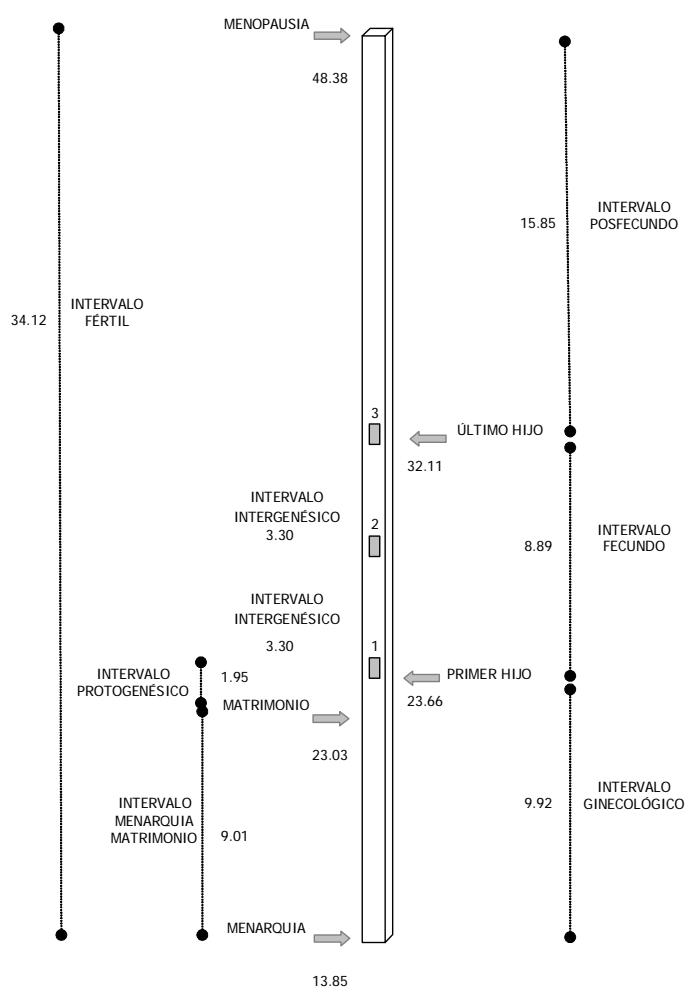

Fig. 1. Historia reproductora media de las mujeres chilenas de Tierra del Fuego. 
mero de hijos vivos más habitual y el rango entre 0 y 11 hijos, con un intervalo intergenésico medio de 3.30 años. Comparado con poblaciones contemporáneas, el valor es inferior al de otras poblaciones en desarrollo, con valores mayoritariamente superiores a los 5 hijos por mujer (a modo de ejemplo: N orth et al., 2000; Crognier et al., 2001) y superior al de las sociedades occidentales actuales. La distribución del número de hijos nacidos vivos de las mujeres de la población muestra, como aspecto más destacable, que las mujeres con 2,3 y 4 hijos nacidos vivos representan el $69.6 \%$ de las mujeres encuestadas en Tierra del Fuego, porcentaje especialmente elevado si lo comparamos con los resultados disponibles de otras poblaciones, donde representan entre el $43.5 \%$ y el $50.2 \%$ (Bertranpetit, 1981 ; Cavalli-Sforza and Bodmer, 1981; Luna, 1981).

La distribución de los nacimientos según la edad de la madre en el momento del nacimiento nos permite conocer cómo varía la fecundidad de las mujeres de una población a lo largo de su vida reproductora. A partir de esta distribución se puede estimar las tasas específicas de fecundidad, y a partir del sumatorio de éstas, la tasa de fecundidad F. Las poblaciones de asentamiento reciente con tasas de fecundidad $F$ superiores a 8 hijos por mujer puede considerarse que a este respecto muestran un patrón de comportamiento reproductor característico de poblaciones colonizadoras; es decir, poblaciones sometidas a una rápida expansión después de la migración a nuevos hábitats que contienen escasos o ningún competidor humano (Wood, 1994). Poblaciones de este tipo serían, por ejemplo, algunas cohortes matrimoniales de Hutteritas, Mennonitas y Amish durante los siglos XVIII, XIX $y X X$, y los canadienses rurales franceses e ingleses durante los primeros años del siglo XVIII. No obstante, otras poblaciones típicamente colonizadoras muestran valores más reducidos: así, por ejemplo, la población australiana en 1921 presenta una tasa de fecundidad de 3.11 y en 1961 de 3.54 (Borrie, 1969). Y, en esta línea, la población chilena de Tierra del Fuego muestra una tasa de fecundidad $F$ también reducida de 3.3 hijos por mujer.

Similares a las tasas específicas de fecundidad, y no siempre distinguidas con rigor, la fecundidad específica para cada edad de las mujeres de una población también puede expresarse mediante las tasas específicas de fecundidad marital, que relacionan los nacimientos de ma- dres de una edad determinada con el total de mujeres casadas a esa edad. A partir de su suma se obtiene para Tierra del Fuego una estima de la tasa de fecundidad marital de 6.43 hijos nacidos vivos por mujer casada. Este valor se encuentra dentro del rango descrito para poblaciones colonizadoras, siendo más bajo que las tasas descritas para poblaciones tradicionales religiosas anabaptistas, con valores entre 7 y 10 hijos nacidos vivos por mujer (Eaton and Mayer, 1953; Cross and McKusick, 1970; Felt et al., 1990; Nonaka et al., 1994) y superior al de otras poblaciones colonizadoras contemporáneas como Australia y Nueva Zelanda, con valores inferiores a 5 hijos por mujer (J ones, 1971; Zodgekar, 1980).

La Figura 2 permite comparar la distribución de las tasas específicas de fecundidad y de fecundidad marital. Se advierte que, para una edad determinada, las tasas de fecundidad marital son mayores que las tasas de fecundidad a lo largo de casi todo el período reproductor de las mujeres, aunque las diferencias mayores se observan en edades menores de 25 años (nótese la diferente escala de ambos ejes de ordenadas).

Aunque la bibliografía sugiere que en las poblaciones donde existe control de la fecundidad, la edad nupcial es más significativa en la definición del calendario de la fecundidad que en el valor de la fecundidad final, se observa en la población fueguina su influencia en ambos aspectos. A su efecto en la fecundidad final ya comentado en el apartado anterior, se añade aquí su influencia en el calendario. Aunque las tasas de máxima fecundidad marital se dan entre los 17 y los 25 años, la edad de acceso al matrimonio condiciona que las tasas específicas de fecundidad máximas se den entre los 24 y los 26 años.

Estos resultados, considerando además la diferencia en los índices sintéticos de fecundidad según provengan de la suma de las tasas específicas de fecundidad (3.3 hijos nacidos vivos por mujer) o de la suma de las tasas específicas de fecundidad marital (6.4 hijos nacidos vivos por mujer), sugieren un papel determinante de la nupcialidad en la fecundidad de la población de Tierra del Fuego, a través de parámetros como la edad de acceso al matrimonio, la ruptura de la unión conyugal o las nupcias repetidas. En esta línea, en Australia se detecta también una importante diferencia entre la tasa de fecundidad $\mathrm{F}$ y la tasa de fecundidad marital, habiéndose descrito para ella la importante influencia que los 


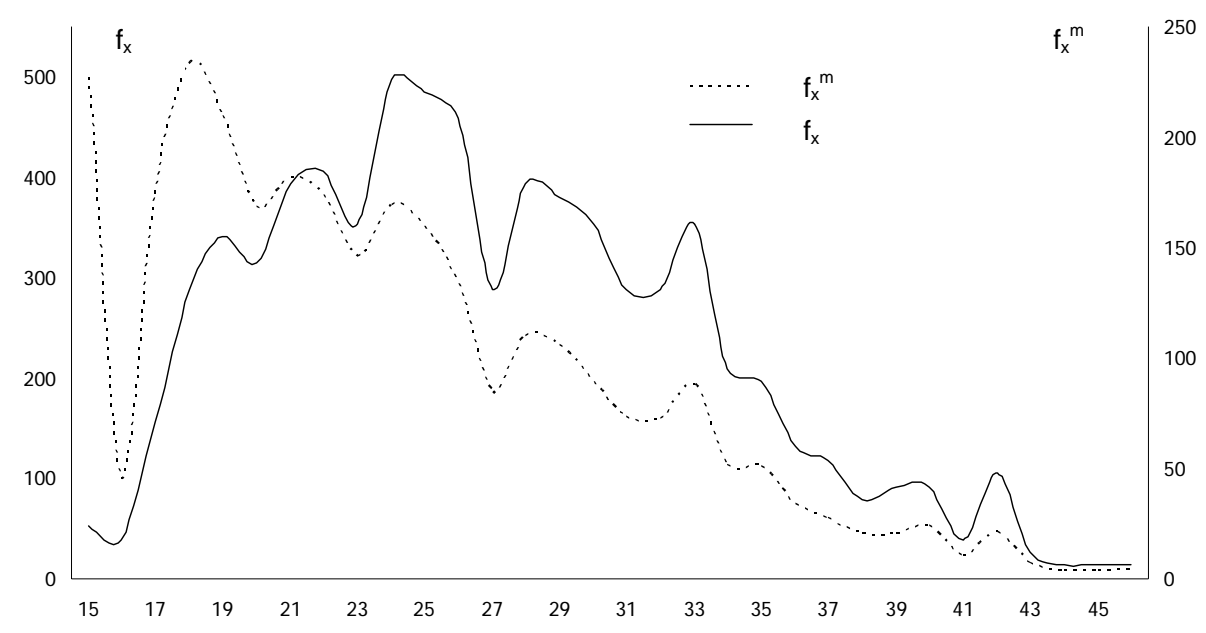

Fig. 2. Distribuciones de las tasas específicas de fecundidad $\left(f_{x}\right)$ y de las tasas específicas de fecundidad marital $\left(f_{x} m\right)$, expresadas como el número de hijos nacidos vivos por 1000 mujeres o mujeres casadas en la población, respectivamente.

patrones nupciales tienen en los niveles de fecundidad de la población (J ones, 1971).

C. Fecundidad y origen de las mujeres.

La población actual de Tierra del Fuego es la resultante de una mezcla biológica y cultural particular originada por la llegada de distintos contingentes inmigrantes. La asincronía de estas corrientes inmigratorias ha establecido un cambio en las características que definen la sociedad fueguina y ello, sin duda, ha tenido una influencia en los patrones reproductores predominantes en la población a lo largo del siglo XX.
En primer lugar, el establecimiento de individuos de muy distintos lugares de origen ha permitido el incremento de la variabilidad genética de la población, aunque este aumento haya quedado potencialmente reducido debido a la elevada endogamia de la población (Hernández et al., 1998). En lo referente al efecto de la endogamia en la fecundidad de las mujeres de la población, se observa además como las parejas endógamas muestran un relativo mayor número medio de hijos que las parejas exógamas (aunque la diferencia no es estadísticamente significativa). También, las parejas endógamas muestran una distribución del tamaño de la progenie

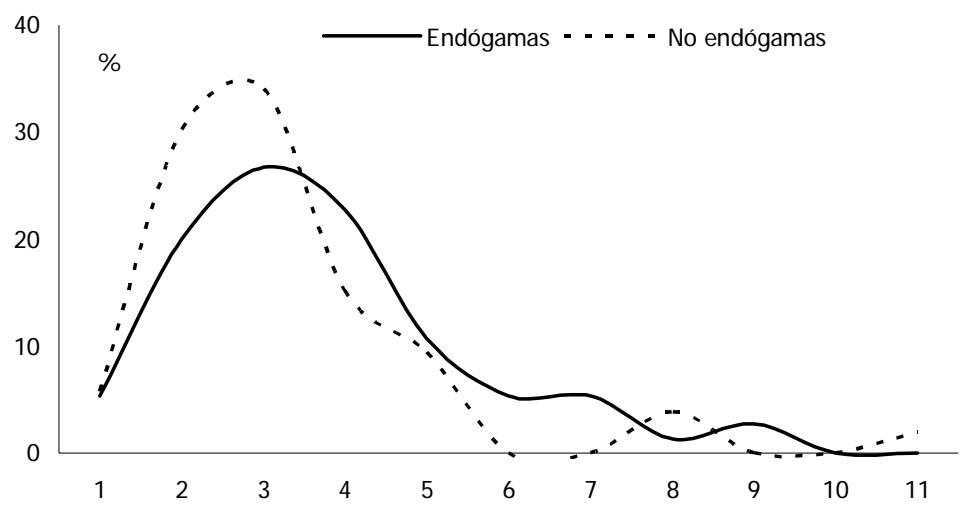

Fig. 3. Distribución del tamaño de la progenie de las parejas endógamas y no endógamas. 
más variable, con un $48 \%$ de las mujeres con más de 3 hijos; en comparación con la distribución más leptocúrtica, es decir, con frecuencias agrupadas en mayor grado alrededor de la media, de las parejas exógamas, con un $30.2 \%$ de parejas con más de 3 hijos (Figura 3).

En segundo término, en lo que se refiere a las diferencias en el calendario de la historia reproductora de las mujeres fueguinas según el origen de éstas, se observan diferencias estadísticamente significativas entre las mujeres chilenas nacidas en Magallanes y las nacidas en otras regiones del país. Las mujeres chilenas de origen magallánico muestran un valor medio menor para las edades medias de menarquia, edad nupcial, y edades de primera y de última maternidad, además de un menor intervalo prenupcial y un mayor intervalo posfecundo, de manera que inician antes su vida reproductora pero también la finalizan antes. Estas diferencias en el calendario reproductor tienen consecuencias en la fecundidad, ya que las mujeres de origen magallánico tienen por término medio un número final de hijos menor, y una menor fecundidad marital, frente a las de otros orígenes, siendo estas diferencias estadísticamente significativas cuando se obtienen los valores a través de reconstrucción de familias y estando cercanas al nivel de significación cuando se calculan a partir de las encuestas de fecundidad (para una información más detallada de las pruebas estadísticas, consultar Pascual, 2004)

En contraposición, las mujeres de origen europeo y las chilenas de origen no magallánico son bastante similares en su calendario reproductor. Las mujeres incluidas en esta última categoría no forman un grupo homogéneo, sino que se observan ciertas diferencias en el calendario de su vida reproductora que después tendrán su influencia en los patrones de reproducción observados. Entre estas últimas, se observa que las mujeres de origen chilote tienen por término medio una menor descendencia y unos intervalos fecundo y posfecundo menores, además de una menor longitud media de los intervalos intergenésicos, que el resto de mujeres chilenas no magallánicas (Pascual, 2004).

Se puede deducir que son las variaciones en el calendario y en la intensidad de los distintos eventos e intervalos de la historia reproductora las que explican mayoritariamente las diferencias observadas en la fecundidad de las mujeres de la población fueguina. Características como el ca- rácter endógamo de la pareja y su lugar de nacimiento permiten interpretar los resultados obtenidos en un contexto histórico y cultural que seguro ha influido en la vida reproductora y en la fecundidad de las mujeres, aunque esta influencia, en ocasiones, no haya sido directa.

D. Tamaño de la progenie y eficiencia de uso de los años reproductores.

Las mujeres fueguinas que tienen un elevado número de hijos utilizan con mayor eficiencia sus años reproductores. Este hecho se comprueba repetidamente a través del análisis de la influencia de diversos eventos e intervalos de la vida reproductora en el tamaño final de la progenie de las mujeres fueguinas.

L a edad nupcial de las mujeres se muestra determinante del número final de hijos que tendrán. Así, el análisis de la varianza del tamaño de la progenie según la edad nupcial de las mujeres muestra una reducción significativa $(F=$ 3.043; g.d.I. 3, 155; $p=0.031$ ) del tamaño medio de la progenie de 3.87 hijos por mujer, para las mujeres menores de 20 años, a los 2.37 hijos nacidos vivos por mujer, de las mujeres mayores de 29 años. Puesto que la edad nupcial se toma en la práctica como punto de referencia del inicio del periodo reproductor de la mujer, los resultados obtenidos indican que el inicio de la vida marital es fundamental en la determinación final del número de hijos de las mujeres encuestadas. En gran medida lo es al condicionar la edad de la madre al nacimiento del primer hijo.

Cuanto más temprana es la edad de primera maternidad mayor es el tamaño medio de la progenie de las mujeres encuestadas 0 , del mismo modo, cuanto más se retrasa la llegada del primer hijo menor es el número medio final de hijos ( $F=6.168 ;$ g.d.I. 3,152; $p=0.001)$. Las mujeres cuya edad de primera maternidad es inferior a los 20 años tienen una media de 4.06 hijos nacidos vivos al final de su periodo reproductor y las que tienen su primer hijo con más de 29 años alcanzan un tamaño medio de 2.37 hijos. Corroborando los resultados descritos para la edad nupcial, el inicio de la vida fecunda condiciona el tamaño de la descendencia final de las mujeres.

En la misma línea que estos resultados previos también se encuentra el análisis de la varianza del número de hijos nacidos vivos según la duración del intervalo ginecológico (el intervalo entre 
la menarquia y la primera maternidad). Cuanto más corto es el intervalo, mayor es el número medio de hijos final $(F=4.234$; g.d.I. 3,135; $p=$ 0.007). Una utilización más eficaz de los primeros años reproductores permite un tamaño de la progenie mayor al final de la vida fértil. Sin embargo, podría no ser sólo una cuestión de eficiencia, en el sentido de que las mujeres que desean tener un mayor número de hijos inician su periodo fecundo con mayor rapidez.

No sólo el inicio, sino también el final del intervalo fecundo es determinante del tamaño de la progenie de las mujeres encuestadas en Tierra del Fuego. El análisis de la varianza del número de hijos nacidos vivos según la edad materna al nacimiento del último hijo indica que existen diferencias significativas $(F=3.769$; g.d.I. 4,150; $p=0.006$ ) entre los subgrupos considerados. Así, las mujeres que tienen su último hijo a edades tempranas (mujeres con edades de última maternidad inferiores a los 25 años) tienen significativamente un menor número de hijos (2.68) que las que muestran edades tardías de última maternidad (edad de última maternidad igual o superior a los 40 años, y tamaño medio de la progenie de 4.50 hijos nacidos vivos).

Finalmente, se constata que el espaciamiento de la descendencia tiene relación también con el tamaño final de la descendencia de las mujeres ( $F=6.778 ;$ g.d.I. 3,$138 ; p=0.000)$, de manera que cuanto mayor es el número de hijos nacidos vivos más corto es el intervalo intergenésico medio (intervalo estimado a partir del promedio de sus distintos intervalos intergenésicos válidos). La reducción que experimenta el intervalo intergenésico medio entre los subgrupos considerados extremos (mujeres con 2 hijos nacidos vivos, con una longitud media del intervalo de 4.67 años; mujeres con más de 4 hijos con una longitud media de 2.42 años) es de 2.2 años, lo cual supone una disminución importante, de casi el $50 \%$, respecto a la longitud del intervalo intergenésico medio mayor. En otras palabras, las mujeres que tienen un reducido número de hijos nacidos vivos muestran una gran heterogeneidad para la longitud de su intervalo intergenésico medio; en cambio, las mujeres con un tamaño de la progenie elevado tienen un comportamiento muy similar, con un intervalo intergenésico medio mucho más pequeño.
E. Indicadores biodemográficos del control de la fecundidad en la población.

Cabe preguntarse si la población de Tierra del Fuego es una población de fecundidad natural sin control de la natalidad o, por el contrario, es de fecundidad controlada; la respuesta es que se trata de una población de fecundidad controlada. No obstante, nuestro interés reside en valorar, por un lado, si la transición de una fecundidad natural a una controlada se dio en algún momento del período en estudio y, por otro, qué parámetros e indicadores biodemográficos dan cuenta de este control de la fecundidad.

De hecho, los valores de fecundidad de la población fueguina no han permanecido invariables a lo largo de todo el siglo XX. Por el contrario, se constata la existencia de una transición de la fecundidad de valores elevados a principios del siglo a unos reducidos en los años más cercanos a la actualidad. En este sentido apunta, por un lado, una medida indirecta de la fecundidad como es la tasa de fecundidad general (en la cual el número de nacimientos vivos anuales se refiere a las mujeres en edad reproductora), que señala que la fecundidad a principios de siglo era bastante elevada (siendo de 263.16 nacimientos por cada 1000 mujeres en edad reproductora en 1906, en contraposición a los 51.05 nacimientos en 1992). Por otro lado, el análisis de la varianza del tamaño de la progenie según el año de nacimiento de las mujeres encuestadas muestra una reducción significativa ( $F=2.639$; g.d.I. 4, 163; $p=0.036$ ) del número de hijos (4.31 por término medio para las mujeres nacidas antes de 1920 y 2.57 hijos para las mujeres en edad posreproductora nacidas a partir de 1950). En este contexto, a pesar de la elevada variabilidad del tamaño final de la progenie entre poblaciones, el número medio de hijos por mujer de la población de Tierra del Fuego se encuentra en el rango de distribución de las poblaciones de fecundidad controlada (Campbell and Wood, 1988).

Además, tanto la reducción a lo largo del tiempo de la variabilidad del número final de hijos como de la presencia de familias numerosas indican una evolución temporal hacia la uniformidad del comportamiento reproductor mediante el control de la fecundidad (Bodmer and J acquard, 1968) (Figura 4). En este sentido, la similitud entre media y varianza de la distribución del tamaño de la progenie (media de 3.32 


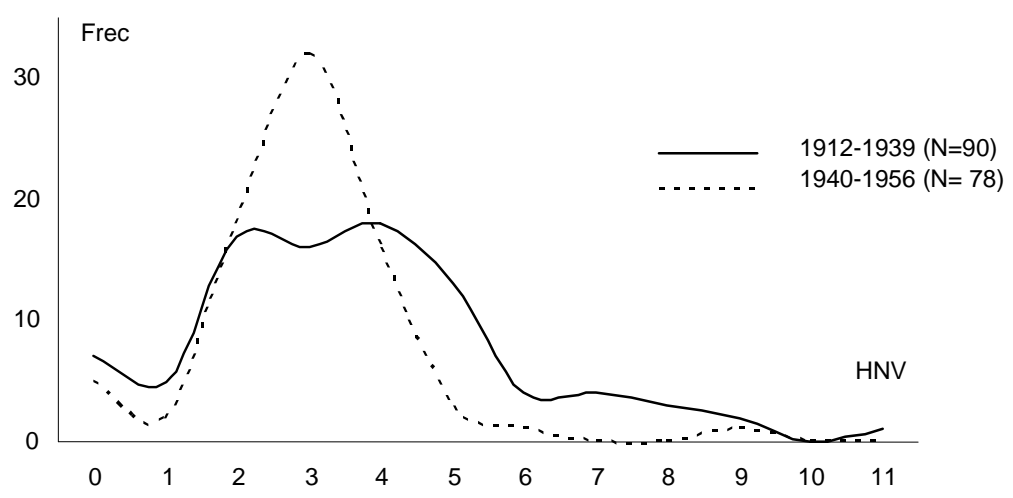

Fig. 4. Evolución temporal de las distribuciones del tamaño final de la progenie según el año de nacimiento de las mujeres. HNV: número de hijos nacidos vivos

hijos y varianza de 3.64), también puede ser un indicador de una cierta planificación familiar, ya que en la mayoría de poblaciones la varianza del tamaño de la progenie es superior a la media entre 1.5 y 3 veces (Cavalli-Sforza and Bodmer, 1981).

Entre los indicadores del control de la fecundidad en la población fueguina, uno de los más directos es la constatación del uso reconocido de prácticas anticonceptivas de alta eficacia en algún momento de su vida reproductora por parte del $73 \%$ de las mujeres. No desear un nuevo embarazo puede responder a la aspiración de espaciar los nacimientos o a la pretensión de limitar el tamaño familiar (Fort, 1989). Sin embargo, la consideración del uso o no de métodos anticonceptivos entre las mujeres encuestadas de Tierra de Fuego no supone diferencias significativas en el número de hijos nacidos vivos ( $t=$ 0.614 , g.d.l.154, $p=0.540$ ). Pese a ello, las me- didas de dispersión de la distribución reflejan una menor variación en el tamaño de la progenie en el grupo de mujeres con descendencia que utilizan algún método anticonceptivo, donde el tamaño de la progenie más frecuente es de 3 hijos nacidos vivos (Figura 5). Ello sugiere la utilización de métodos anticonceptivos como un medio para alcanzar el tamaño familiar deseado.

Con la intención de buscar otros indicadores del control de la fecundidad en las mujeres fueguinas, nuestro interés se centra en el análisis de las tasas de fecundidad. La comparación de las tasas específicas de fecundidad marital de diversas poblaciones históricas europeas de fecundidad natural muestra que los niveles son muy variables; no obstante, si las referimos to das a la tasa específica del grupo de edad de 20-24 años (a la que le asignamos el valor de 100), se observa que los patrones de fecundidad por edad

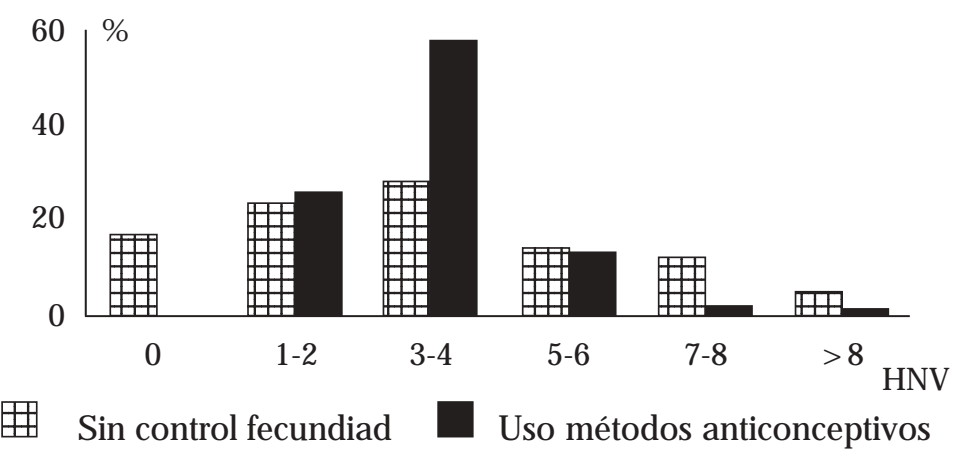

Fig. 5. Distribución de las frecuencias del tamaño de la progenie según el uso o no de métodos anticonceptivos para mujeres con descendencia. 


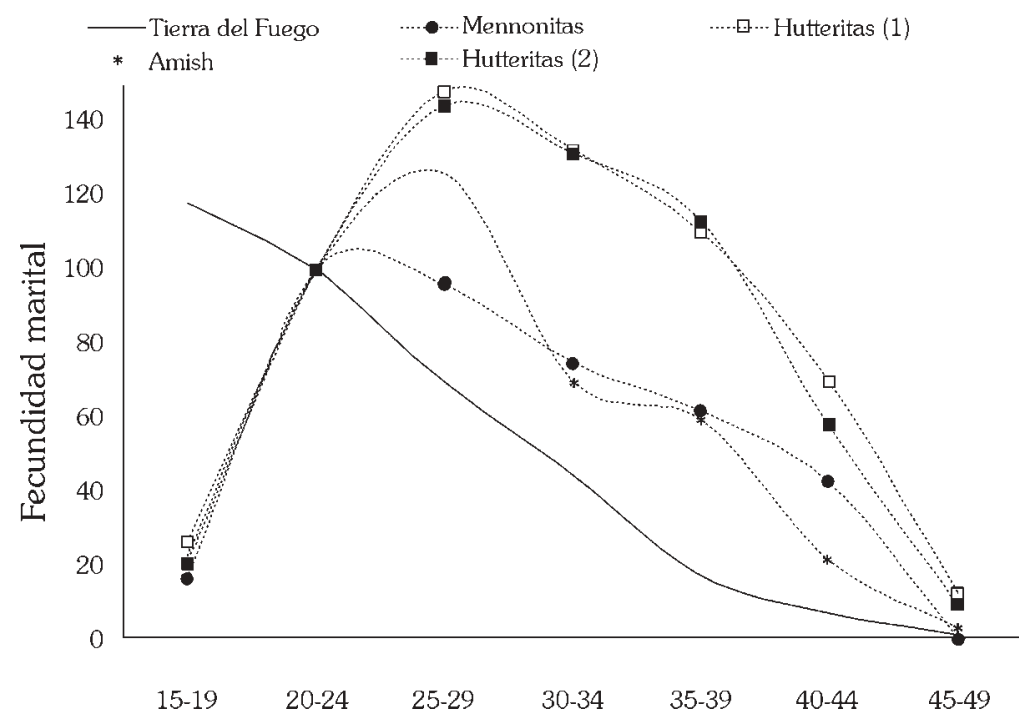

Fig. 6. Patrones de fecundidad por grupos de edad de poblaciones de fecundidad natural y de la población de Tierra del Fuego. Datos tomados de: Mennonitas (Felt et al., 1990); Hutteritas (1) (Eaton and Mayer, 1953); Amish (G reksa, 2002); Hutteritas (2) (Nonaka et al., 1994).

de las poblaciones con fecundidad natural son básicamente idénticos, aunque los niveles varíen considerablemente (Henry, 1961; Coale and Trussell, 1974; Bongaarts and Potter, 1983; Smith, 1988). Además, este patrón común es distinto del que muestran las poblaciones que controlan su natalidad. En una población de fecundidad (o de fecundidad marital) natural y colonizadora se esperaría una curva de forma convexa; no obstante, la curva que muestran las mujeres encuestadas de Tierra del Fuego no presenta convexidad (tanto la que representa el patrón de tasas específicas de fecundidad como la de las tasas específicas de fecundidad marital), tal como se esperaría para una población de fecundidad controlada (Figura 6). De la misma manera, la magnitud de las tasas sitúa, de nuevo, a la población fueguina entre poblaciones de baja fecundidad, aunque por encima de las tasas que muestran las poblaciones occidentales más desarrolladas (Bongaarts and Potter, 1983).

Los datos de la Tabla 1 completan los mostrados en la Figura 6. El patrón que se observa en las mujeres fueguinas se aleja de lo esperado para una población que presenta fecundidad natural, de manera que la probabilidad de tener un hijo es especialmente reducida en los últimos años de la vida reproductora; además, las edades más

TABLA 1. Tasas específicas de fecundidad $\left(f_{x}\right)$ y de fecundidad marital $\left(f_{x}{ }^{m}\right)$ de las mujeres por grupos de edad, expresadas como el número de hijos por cada 1000 mujeres. Las tasas de fecundidad $F$ y de fecundidad marital $\mathrm{F}^{\mathrm{m}}$ expresan el número de hijos por mujer.

\begin{tabular}{|c|r|c|c|c|}
\hline Edad de la madre & $\mathrm{f}_{\mathrm{x}}$ & $\mathrm{f}_{\mathrm{x}} / \mathrm{f}_{20-24} * 100$ & $\mathrm{f}_{\mathrm{x}} \mathrm{m}$ & $\mathrm{f}_{\mathrm{x}} \mathrm{m} / \mathrm{f}_{20-24} \mathrm{~m} * 100$ \\
\hline $15-19$ & 79.8 & 44.7 & 435.1 & 118.2 \\
\hline $20-24$ & 178.6 & 100.0 & 368.0 & 100.0 \\
\hline $25-29$ & 182.1 & 102.0 & 259.1 & 70.4 \\
\hline $30-34$ & 135.7 & 76.0 & 164.6 & 44.7 \\
\hline $35-39$ & 56.0 & 31.3 & 67.3 & 18.3 \\
\hline $40-44$ & 25.0 & 14.0 & 29.5 & 8.0 \\
\hline $45-49$ & 2.4 & 3.3 & 8.4 & 2.3 \\
\hline$F$ & 3.3 & $F^{m}$ & 6.6 & \\
\hline
\end{tabular}




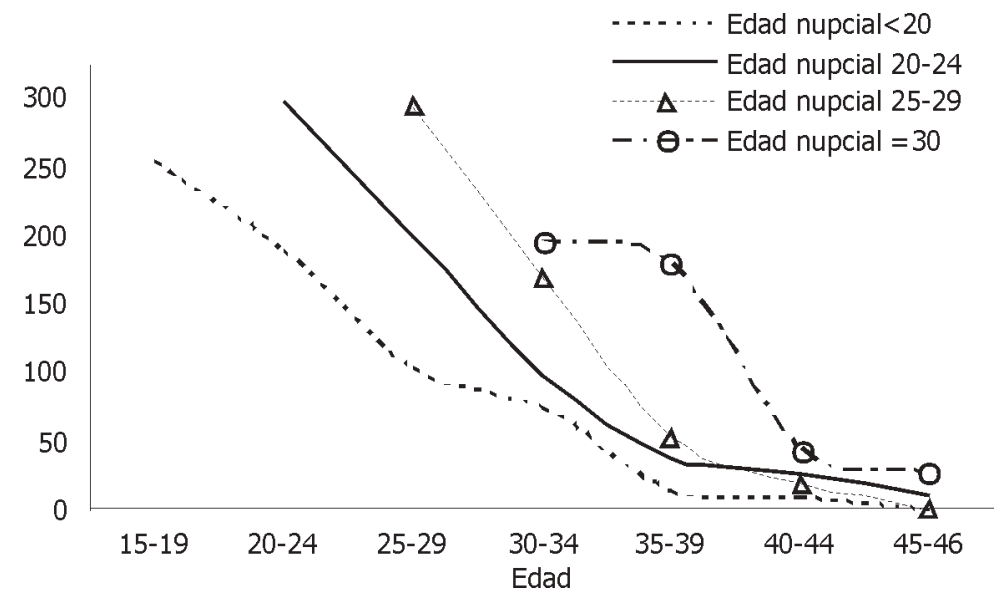

Fig. 7. Tasas específicas de fecundidad marital según la edad nupcial de las mujeres.

avanzadas tienen escasa importancia en el tamaño final de la descendencia. También es interesante observar que la tasa específica de fecundidad para el grupo de edad 25-29 años es mayor que la del grupo de edad 20-24 años, pero no ocurre lo mismo si lo que se compara son las tasas específicas de fecundidad marital. El patrón en Tierra del Fuego viene determinado por el patrón de nupcialidad, de manera que la entrada al matrimonio modifica la tendencia esperable en la cual la tasa del grupo 20-24 debiera ser la mayor.

Otra diferencia entre las poblaciones de fecundidad natural y las que la controlan se hace evidente cuando los datos de fecundidad marital se clasifican conjuntamente según la edad de la madre y su edad nupcial. En el caso de una población de fecundidad natural, las curvas resultantes coinciden ampliamente y no difieren de manera clara. En contraposición, en condiciones de fecundidad controlada, las tasas de fecundidad marital se escalonan según la duración del matrimonio, de manera que las tasas para mayores duraciones (mujeres casadas en edades más tempranas) disminuyen más tempranamente que aquellas para duraciones más cortas (Wood, 1994). Los resultados representados en la figura 7 corroboran la relación positiva entre la tasa de fecundidad marital y la edad nupcial y, de nuevo, ponen de manifiesto la eficacia del control de natalidad en la población fueguina.

Centrándonos en otro evento de la vida reproductora de las mujeres, el aumento del último ( $\mathrm{y}$, a veces, también penúltimo) intervalo intergenésico cerrado, independiente de su paridad, ha sido descrito en la bibliografía como indicador de que existe control de natalidad en la población. De esta manera, la decisión de limitar la fecundidad sucedería solamente después de haber alcanzado el tamaño de la descendencia deseado: la adopción del control de la fecundidad sería dependiente de la paridad (Anderton and Bean, 1985). En Tierra del Fuego, la longitud del intervalo intergenésico es siempre mayor si el hijo es el último de la mujer sea cual sea el número de orden del nacimiento. Sin embargo, las diferencias entre las longitudes medias son solamente significativas en las paridades más bajas ( 2 y 3, estando cercano al límite de significación para la paridad 4), donde son mayores las diferencias observadas (más de 2 años) (Pascual, 2004). En mujeres con tamaños de la descendencia superiores, es menos probable que exista un interés por limitar la fecundidad.

A la vista de los resultados aquí presentados, es evidente el control de la fecundidad por parte de un importante sector de las mujeres fueguinas. Sin embargo, nos interesa discernir si esta regulación de la fecundidad tiene como objetivo principal limitar la fecundidad de las mujeres (stopping behaviour) y/o adaptar el espaciamiento de los hijos a intereses o necesidades de la familia (spacing behaviour). En este sentido, el análisis de los intervalos intergenésicos permite afirmar que el mayor espaciamiento intencionado de los distintos nacimientos es posible visto el efecto de elongación estadísticamente significativo que el uso de los métodos anticon- 
ceptivos tiene en la longitud de los intervalos, efecto que se mantiene en las distintas paridades. También en esta línea apunta el efecto alargador que el uso de métodos anticonceptivos tiene en la longitud del intervalo fecundo, de manera que, por término medio, el intervalo fecundo es dos años menor en las mujeres que controlaron su fecundidad.

En contraposición, existen también diversos indicadores de que aparece un comportamiento limitador de la fecundidad en las mujeres de la población, lo que reflejaría el interés de muchas mujeres por alcanzar un número de hijos deseado que determinaría el final del perío do fecundo. Entre ellos, la importancia del tamaño de la progenie y del cierre de la descendencia en la longitud del intervalo intergenésico, así como la reducción de la variabilidad en el tamaño de la progenie y en la temprana edad de última maternidad asociada al uso de métodos anticonceptivos, el aumento del tiempo no destinado a tener descendencia a lo largo del período estudiado y la adopción de la esterilización definitiva voluntaria en mujeres con intervalos intergenésicos medios pequeños. De hecho, el espaciamiento de los hijos parece representar una estrategia adicional para limitar la fecundidad de una población (Anderton and Bean, 1985) y así parecen indicarlo los resultados del análisis aquí presentados.

F. Determinantes de la fecundidad. Modelo agregativo de Bongaarts y Potter.

Solamente aquellas características que intervienen directamente en algún proceso biológico pueden determinar efectivamente la fecundidad de las mujeres de una población. Estas variables relacionadas directamente se denominan determinantes próximos de la fecundidad, siendo las restantes determinantes distantes de la fecundidad.

Bongaarts (1978) redujo la lista de los determinantes próximos a ocho, creando un marco de referencia para el análisis de la fecundidad, comúnmente aceptado en la actualidad. Los determinantes próximos de la fecundidad (Bongaarts, 1978; Wood, 1994) son: (1) la proporción de mujeres casadas (que permite estimar la proporción de mujeres de edad reproductora que tiene relaciones sexuales de manera regular); (2) la anticoncepción (que incluye cualquier práctica intencionada dependiente de la paridad -incluidas la abstención y la esterilización- con el fin de reducir el riesgo de concepción); (3) la inducción del aborto (considerando aquí cualquier práctica que deliberadamente interrumpe el curso normal de gestación); (4) la esterilidad por lactancia (período temporal posterior al parto en el que la mujer es incapaz de concebir hasta que se restaura el patrón normal de ovulación y menstruación, y que depende principalmente de la duración e intensidad de las prácticas de lactancia materna); (5) la frecuencia de relaciones sexuales (que determina la fecundabilidad natural de una población); (6) la esterilidad de las mujeres; (7) la mortalidad intrauterina espontánea (que evita que todas las concepciones den lugar a un nacido vivo); y (8) la duración del período fértil (que tiene en cuenta que una mujer es capaz de concebir solamente durante un período breve en su ciclo menstrual).

El propósito del presente apartado es la cuantificación de la importancia de los determinantes próximos de la fecundidad en las mujeres de la población chilena de Tierra del Fuego, mediante un modelo de fecundidad. El modelo agregativo de la fecundidad propuesto por Bongaarts $(1978,1984,1987,1994)$ y Bongaarts and Potter (1983) es una aproximación válida a la descomposición de las contribuciones de los diferentes determinantes próximos a los niveles y tendencias de fecundidad observados en la población. Discriminando entre los determinantes próximos aquellos que muestran poca variación en el tiempo o entre poblaciones, y los que no modifican de manera significativa la fecundidad, el modelo se detiene especialmente en el análisis del papel de cuatro determinantes que explican la mayor parte de la variabilidad obser-

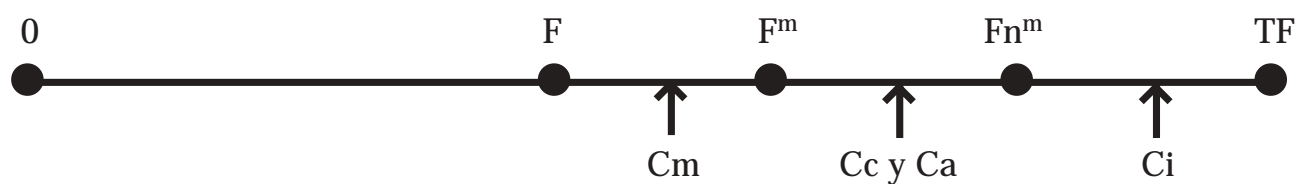

Fig. 8. Relación entre los efectos inhibidores de los determinantes próximos y varias medidas de fecundidad. El significado de los distintos parámetros y su definición quedan reflejados en el texto. 
TABLA 2. Cuantificación del efecto inhibidor de cuatro determinantes próximos en la fecundidad de la población fueguina.

\begin{tabular}{|lcc|}
\hline Índices calculados & Estimas obtenidas & Reducción de la fecundidad \\
\hline Índice nupcial & 0.495 & $50 \%$ \\
Índice de anticoncepción & 0.445 & $56 \%$ \\
Índice de aborto inducido & 1 & $0 \%$ \\
Índice de esterilidad posparto & 0.778 & $22 \%$ \\
\hline
\end{tabular}

vada en la fecundidad de las poblaciones humanas: la nupcialidad (como medida de exposición a las relaciones sexuales), las prácticas anticonceptivas y la duración de la esterilidad posparto (como medidas de exposición a la concepción) y la inducción del aborto (como medida de exposición al parto). De hecho, en un análisis de la fecundidad de 41 poblaciones, el modelo explica el $96 \%$ de la variabilidad observada en la fecundidad entre poblaciones (Bongaarts and Potter, 1983) y en otro estudio de 29 poblaciones en desarrollo explica el $71 \%$ de la variabilidad observada (Cleland et al., 1984).

Este modelo agregativo distingue cuatro niveles de fecundidad distintos, derivado cada uno de ellos de la acción de un determinante próximo, siendo así la tasa de fecundidad $\mathrm{F}$ el resultado de la acción inhibidora de todos los determinantes próximos (Figura 8). Al mismo tiempo, los efectos que sobre la fecundidad ejercen los cuatro determinantes próximos incluidos en el modelo son cuantificados mediante cuatro índices: el índice nupcial ( $\mathrm{Cm})$, el índice de anticoncepción (Cc), el índice de aborto inducido (Ca) y el índice de esterilidad posparto ( $\mathrm{Ci}$ ). Los índices toman valores entre 0 y 1 . Cuando no existe ningún efecto inhibidor, el índice vale 1 ; si la inhibición es plena, el índice es igual a cero.

Si se elimina el efecto que los patrones de nupcialidad tienen en la fecundidad, ésta aumenta hasta el nivel de la tasa de fecundidad marital $\left(\mathrm{F}^{\mathrm{m}}\right)$. En la misma línea, si se elimina el efecto de las prácticas anticonceptivas y de la inducción del aborto en la fecundidad, ésta aumenta hasta el nivel de la tasa de fecundidad natural marital $\left(\mathrm{Fn}^{\mathrm{m}}\right)$. Finalmente, si además, se elimina el efecto que en la fecundidad tienen las prácticas de lactancia materna y de abstinencia sexual después del parto la fecundidad se incrementa hasta la tasa global de fertilidad (TF). Esta tasa global de fertilidad representa la descendencia final teórica en ausencia de variables que limiten la fe- cundidad de las mujeres de la población. En el caso hipotético de ausencia de determinantes próximos de la fecundidad en una población humana, su fecundidad estaría determinada por la capacidad biológica de concebir de las mujeres, es decir, por su fertilidad.

La Tabla 2 resume los índices obtenidos para los diversos determinantes próximos de la fecundidad en la población fueguina. La edad nupcial relativamente tardía, las proporciones de mujeres casadas relativamente bajas y las tasas de viudedad y ruptura conyugal con unos porcentajes intermedios entre los observados en otras poblaciones sugieren un importante papel del patrón de nupcialidad en la determinación de la fecundidad de las mujeres de Tierra del Fuego. El índice de nupcialidad del modelo indica que contribuyen a reducir en un $50 \%$ la fecundidad potencial de la población.

En la misma línea, el índice de anticoncepción muestra que el control de la fecundidad es una práctica relativamente extendida entre las mujeres encuestadas de la población de Tierra del Fuego, lo cual confiere a la anticoncepción, a la eficiencia de su uso y a las prácticas de esterilización definitiva un papel importante en la descendencia final de las mujeres encuestadas, reduciendo en un $55 \%$ la fecundidad potencial de la población. El 90\% de las mujeres con 3 hijos, las más representadas en la distribución de frecuencias del tamaño de la progenie, controlan su fecundidad. Como técnica anticonceptiva entre las mujeres de Tierra del Fuego aparece con cierta importancia la esterilización definitiva, asociada mayoritariamente a tamaños de la progenie de 3 y 4 hijos por mujer y, con mayor frecuencia, en mujeres con intervalos intergenésicos medios pequeños, lo que sugeriría que es adoptada como solución por mujeres que han tenido diversos hijos con relativa rapidez.

Por otro lado, la inducción del aborto como método para controlar la natalidad de la población parece una práctica aislada en Tierra del 
TABLA 3. Grados de determinación de las variables consideradas en los modelos 1 y 2 . EMN 1: edad de primera maternidad; IFC: intervalo fecundo de las mujeres; EMNu: edad de última maternidad; HNV: número de hijos nacidos vivos; Anticon: años de uso de anticonceptivos. IIM: intervalo intergenésico medio. Esteril: edad del cese definitivo fertilidad.

\begin{tabular}{|c|c|c|c|c|c|}
\hline \multicolumn{2}{|c|}{ Determinación } & Directa & Combinada & Total & No explicada \\
\hline EMN 1 & $\begin{array}{l}\text { Edad menarquia } \\
\text { Edad nupcial }\end{array}$ & $\begin{array}{l}0.051 \\
0.766\end{array}$ & -0.043 & 0.774 & 0.226 \\
\hline EMNu & $\begin{array}{l}\text { EMN } 1 \\
\text { IIM }\end{array}$ & $\begin{array}{l}0.264 \\
0.235\end{array}$ & 0.014 & 0.513 & 0.487 \\
\hline IFC & $\begin{array}{l}\text { Anticon } \\
\text { EMNu }\end{array}$ & $\begin{array}{l}0.043 \\
0.471\end{array}$ & -0.079 & 0.435 & 0.565 \\
\hline \multicolumn{6}{|l|}{ Modelo 1} \\
\hline Embarazos & $\begin{array}{l}\text { Edad Nupcial } \\
\text { IFC }\end{array}$ & $\begin{array}{l}0.138 \\
0.331\end{array}$ & -0.092 & 0.377 & 0.623 \\
\hline \multicolumn{6}{|l|}{ Modelo 2} \\
\hline Embarazos & $\begin{array}{l}\text { Edad Nupcial } \\
\text { Años mat. } \\
\text { IFC }\end{array}$ & $\begin{array}{l}0.107 \\
0.042 \\
0.352\end{array}$ & -0.111 & 0.389 & 0.611 \\
\hline
\end{tabular}

Fuego, aunque la existencia de abortos espontáneos no es infrecuente en las historias reproductoras de las mujeres encuestadas. Finalmente, la estima del índice de esterilidad posparto indica que las prácticas de lactancia materna y/o la abstinencia posparto de las mujeres fueguinas influyen poco en la fecundidad de la población reduciendo su fecundidad potencial en un $22 \%$.

El análisis de la evolución temporal de los diversos índices muestra un cambio del papel de los determinantes próximos en la fecundidad de Tierra del Fuego. Así, además de una reducción de la tasa de fecundidad marital en la población, se observa un incremento importante del efecto inhibidor de las prácticas anticonceptivas, que coincide con una extensión de las prácticas anticonceptivas en la población a medida que transcurre el siglo XX, especialmente a partir de los años sesenta. Los anticonceptivos orales son los más usados por las mujeres encuestadas, aunque la mayor variabilidad en el tamaño de la progenie de las mujeres que los utilizan indica la relativa ineficiencia del uso de este método en la población (Pascual, 2004).

La disponibilidad para un número importante de poblaciones de estimas de los distintos índices considerados en el modelo, permite com- parar los resultados obtenidos para la población fueguina con los de otras poblaciones. En este sentido, a partir de la tasa de fecundidad se suelen distinguir cuatro fases o estadios de la transición de una fecundidad natural a una controlada, en las que la mayoría de las poblaciones en fase I tienen una fecundidad natural o muy próxima a ella, mientras que las poblaciones en fase IV realizan un control importante o total de la natalidad. Cada una de las cuatro fases se caracteriza por unos índices promedio de los determinantes próximos. Los valores obtenidos para la

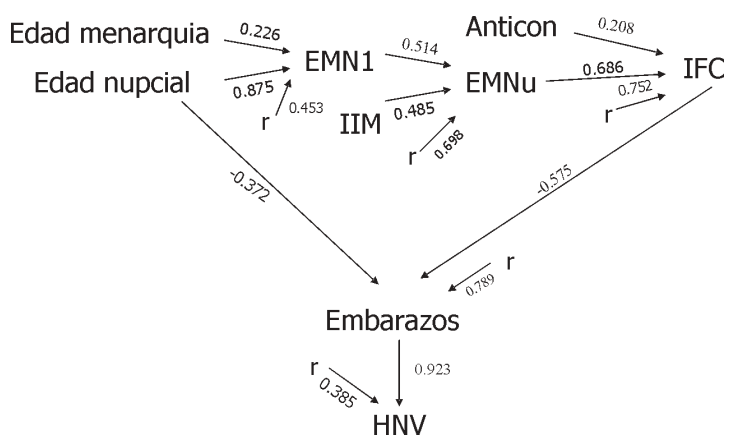

Fig. 9. Diagrama de paths que muestra el modelo 1 que caracteriza las mujeres encuestadas en Tierra del Fuego. 


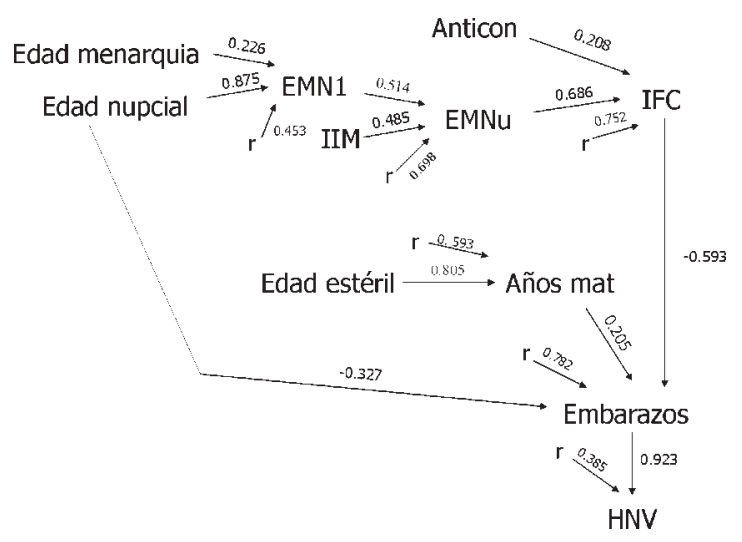

Fig. 10. Diagrama de paths que muestra el modelo 2 que caracteriza a las mujeres encuestadas en Tierra del Fuego.

población fueguina la sitúan en un estadio avanzado de la transición en la fecundidad, en un estadio intermedio entre la fase III y la fase IV.

De esta manera, la transición de la fecundidad en la población, apuntada en un apartado anterior mediante el análisis de diversas medidas de fecundidad, se constata también a través de la cuantificación de la importancia de diversos determinantes próximos de la fecundidad. La población fueguina pasa de un estado de escaso control de la fecundidad a principios del siglo XX a una fase de reducida fecundidad ya en la segunda mitad del siglo XX, lo que contrasta con lo observado para el resto del país chileno y otras poblaciones de América Latina (Bongaarts and Potter, 1983; Cleland et al., 1984).

\section{G. Determinantes de la fecundidad. Path analysis.}

Una aproximación distinta al análisis de la importancia de los distintos determinantes de la fecundidad en la población de Tierra del Fuego consiste en ahondar en las interconexiones entre las variables que se considera que influyen en los patrones reproductores observados. En este sentido, el método de path analysis permite descomponer los coeficientes de correlación que caracterizan las interconexiones entre variables a partir de unas estructuras preconcebidas que definen las relaciones entre éstas (para profundizar en la base teórica del método o en aspectos más prácticos consultar Li, 1975; Pascual et al., 2005). A partir de las correlaciones existentes entre las variables consideradas en el modelo, y en base a los diagramas representados en las Figuras 9 y
10 , se estimaron los coeficientes de path correspondientes que cuantifican las relaciones sugeridas (también incluidos en las figuras y en la tabla 3). Entre la diversidad de modelos explicativos de las interrelaciones entre las variables consideradas se han seleccionado dos en base a las conexiones descritas por otros trabajos consultados y en los considerables porcentajes de variabilidad observada explicados. Los modelos presentados son similares y se diferencian solamente en el tramo final de los diagramas (Pascual et al., 2005).

L a importancia de los patrones de nupcialidad y de anticoncepción señalados mediante el modelo agregativo de la fecundidad se corrobora también a partir del path analysis. La entrada en la vida conyugal, el ritmo de concepción y la edad de última maternidad determinan mayoritariamente el número de embarazos que experimentará una mujer, y la variabilidad entre mujeres en estas variables determinará la mayor parte de la variabilidad del número de embarazos (y también de la fecundidad) en las mujeres fueguinas.

El método del path analysis permite, además, profundizar en las interrelaciones entre los diversos eventos e intervalos de la historia reproductora de las mujeres fueguinas. La edad nupcial se muestra claramente como el factor más explicativo de la variabilidad de la edad de primera maternidad. Por otro lado, la edad a la que la mujer inicia su periodo fecundo y el ritmo en la concepción de la descendencia (definido posiblemente en mayor medida por el tiempo de amamantamiento materno, el uso de prácticas anticonceptivas y la separación temporal o definitiva de los cónyuges) determinan de manera positiva la edad a la cual la mujer tendrá su último hijo. La longitud del intervalo fecundo de la mujer depende en mayor medida de la edad de última maternidad, y este punto final depende seguro de parámetros como la pérdida parcial o total de fertilidad de la mujer y el uso de métodos anticonceptivos.

La aplicación del path analysis ha mostrado patrones reproductores distintos entre las poblaciones humanas. En el estudio de la fecundidad en poblaciones de la India Central muestra la duración del matrimonio como el factor más determinante del número de hijos nacidos vivos (Loebner and Driver, 1973), variable considerada a menudo en la bibliografía como determinante de la fecundidad de las poblaciones hu- 
manas (Luna and Fúster, 1990). La importancia de la duración del matrimonio en estas poblaciones es un indicador indirecto de que las prácticas anticonceptivas son poco frecuentes o poco efectivas, de manera que tienen hijos durante toda su vida fértil (Loebner and Driver, 1973). Por otro lado, la edad nupcial también se muestra importante en las poblaciones de la India Central, ya que la capacidad reproductora de la mujer es mayor en sus primeros años de vida fértil (Loebner and Driver, 1973). En cambio, en la población berebere son el intervalo intergenésico medio y el intervalo definido por la edad nupcial y la edad materna en el nacimiento del último hijo las variables más explicativas del número de hijos nacidos vivos (Crognier, 1996).

Las mujeres de la población chilena de Tierra del Fuego muestran un patrón claramente distinto al de las poblaciones de la India Central. La duración del matrimonio ha sido considerada aquí de manera corregida a través del número de años de matrimonio en los que la mujer ha sido fértil. Aunque se muestra explicativa (explica de manera directa el $4.2 \%$ de la variabilidad del número de embarazos), dista de ser la variable determinante del número de embarazos, lo que sugiere el uso en la población de prácticas anticonceptivas. Sin embargo, los resultados obtenidos para Tierra del Fuego son similares a los de la población berebere, puesto que aunque las variables más determinantes son relativamente distintas, son en ambas poblaciones parámetros que reflejan el inicio de la relación conyugal, el ritmo de concepción y el cese del período fecundo. De este modo, la aproximación al análisis de los determinantes de la fecundidad a través del path analysis muestra un patrón de la fecundidad caracterizado por la importancia de variables de tipo biológico y de tipo sociocultural en la variabilidad de la fecundidad de las mujeres de la población de Tierra del Fuego. Los resultados confirman y complementan los anteriormente obtenidos a través del modelo agregativo de Bongaarts.

En conclusión, el modelo de la fecundidad de Tierra del Fuego no se ajusta a los modelos descritos de poblaciones tradicionales de fecundidad natural como los Amish o los Menonitas (Felt et al., 1990; Hewner, 1998; G reksa, 2002), 0 de poblaciones históricas europeas (Henry, 1961; Knodel and Wilson, 1981). Tampoco se ajusta a poblaciones más próximas geográficamente como son los Mapuches de Río Negro de la Patagonia argentina (Crognier et al., 1996) donde el uso de anticoncepción está ampliamente extendido pero limitado al periodo posterior al alcance del tamaño de la progenie deseado, o donde no hay regularización del acceso a la fecundidad porque el matrimonio no es universal en la población.

En este sentido, las comparaciones con otras poblaciones colonizadoras (Campbell and Word, 1988) muestran que los canadienses durante las primeras décadas del siglo XVIII (Charbonneau et al., 1987), los hutteritas durante las últimas décadas del siglo XIX e inicios del XX (Eaton and Mayer, 1953), los mormones durante su expansión en el siglo XIX (Mineau et al., 1979) y la población colonizadora de California (G arcía-Moro et al., 1997) comparten los principales determinantes de la fecundidad descritos para poblaciones de fecundidad natural. Entre estos determinantes destacan el inicio de la vida conyugal y la duración de la misma, además de la disolución del matrimonio antes del final del período reproductor y la ausencia de anticoncepción. En contraposición, la fecundidad de las mujeres fueguinas está condicionada por diversos factores que la limitan y que actúan a través de los determinantes próximos. Fundamentalmente, los patrones de nupcialidad (la relativamente tardía entrada al matrimonio, la separación y viudedad tempranas y la incidencia del celibato definitivo) y el uso extendido de técnicas anticonceptivas modernas, aunque también las características que definen el patrón de espaciamiento de la descendencia, son esenciales para explicar los niveles de fecundidad alcanzados por la población fueguina.

\section{CONCLUSIONES}

a) El patrón reproductor medio de las mujeres fueguinas puede resumirse en las siguientes características: un elevado intervalo fértil teórico de las mujeres (34.12 años), definido por unas edades medias de menarquia (13.85 años) y de menopausia (48.38 años) intermedias entre los rangos descritos para otras poblaciones. La edad nupcial, relativamente tardía (23.03 años), condiciona la edad de primera maternidad (23.66 años) y los intervalos previos a ésta, especialmente el intervalo que transcurre entre la menarquia y el matrimonio (9.01 años) y el intervalo ginecológico (9.92 años). El intervalo protogenésico medio (1.95 años) es elevado. Las mujeres fuegui- 
nas destinan a tener descendencia únicamente una reducida fracción de su intervalo fértil $(8.89$ años). El tamaño medio de la progenie es reducido, de 3.3 hijos por mujer, con los hijos espaciados por unos intervalos intergenésicos largos (en promedio de 3.30 años). La temprana edad media de última maternidad (32.11 años) comporta también una longitud media elevada del intervalo posfecundo (15.85 años).

b) Las mujeres de Tierra del Fuego regulan su fecundidad limitándola (stopping behaviour) y espaciando su descendencia (spacing behaviour). L a edad de entrada en la vida conyugal, el ritmo de concepción y la edad de última maternidad determinan mayoritariamente el número de embarazos que experimentará una mujer.

c) La aplicación del modelo agregativo de la fecundidad de Bongaarts y Potter y del path analysis muestra que la fecundidad de las mujeres fueguinas está condicionada por diversos factores que la limitan y que actúan a través de los determinantes próximos. Fundamentalmente, los patrones de nupcialidad (la relativamente tardía entrada al matrimonio, la separación y viudedad tempranas y la incidencia del celibato definitivo) y el uso extendido de prácticas anticonceptivas modernas, aunque también las características que definen el patrón de espaciamiento de la descendencia, son esenciales para explicar los niveles de fecundidad alcanzados por la población fueguina.

d) La población chilena de Tierra del Fuego se encuentra en un estadio intermedio entre las fases III y IV de la transición de una fecundidad natural a otra controlada; si bien, los valores de distintos índices la sitúan más cercana a la fase III. Este proceso se habría producido con anterioridad al del conjunto del país chileno y, posiblemente, haya coincidido en el tiempo con la transición de la fecundidad en otros países del cono sur americano.

\section{AGRADECIMIENTOS}

Q ueremos expresar nuestro agradecimiento a los representantes de las distintas instituciones que nos atendieron durante nuestras visitas de trabajo a Punta Arenas y Tierra del Fuego; así como a las personas que, a título individual o en representación de asociaciones o entidades, nos apoyaron durante las diversas campañas de recolección de datos. También deseamos agradecer de manera muy especial su colaboración a todas aquellas mujeres que abrieron las puertas de sus hogares y de sus vidas para poder realizar este trabajo.

\section{BIBLIOGRAFÍA}

ANDERTON, D.L. AND BEAN, L.L. 1985. Birth spacing and fertility limitation: a behavioral analysis of a nineteenth century frontier population. Demography 22(2): 169-83.

BERTRANPETIT, J . 1981. Estructura demográfica y genética de la población de Formentera. Tesis doctoral. Universitat de Barcelona. /MS.

BODMER, W.F. AND J ACQUARD, A. 1968. La variance de la dimension des familles. Population 23:869-878.

BONGAARTS, J. 1978. A framework for analyzing the proximate determinants of fertility. Population and Development Review 4(1): 105-132.

BONGAARTS, J. AND POTTER, R. 1983. Fertility, biology, and behavior. An analysis of the proximate determinants. Academic Press. New York.

BONGAARTS, J .; FRANK, O.; LESTHAEGE, R. 1984. The proximate determinants of fertility in sub-Saharan Africa. Population and Development Review 10(3): 511-38.

BONGAARTS, J. 1987. The proximate determinants of exceptionally high fertility. Population and Development Review 13(1): 133-40.

BONGAARTS, J . 1994. The impact of the proximate determinants of fertility: a comment on Reinis. Population Studies 48:159-60.

BORRIE, W. 1969. Recent trends and patterns in fertility in Australia. J ournal of Biosocial Science 1(1): 57-70.

CAMPBELL, K.L. AND WOOD, J.W. 1988. Fertility in traditional societies. Natural human fertility. Social and Biological determinants 39-69. Diggory, P. et al.(eds). MacMillan Press and The Eugenics Society. London.

CAVALLI-SFORZA, L.L. AND BODMER, W.F. 1981. Genética de las poblaciones humanas. Ediciones Omega. Barcelona.

CHARBONNEAU, H.; DESJARDINS, B.; GUILLEMETTE, A.; LANDRY, Y.; LEGARE, J .; NAULT, F. 1987. Naissance d'une population. Les Français établis au Canada au XVIIe siècle. Institut National d'Études Démographiques. Presses de I'Université de Montréal.

CLELAND, J .; CASTERLINE, J .B.; SINGH, S.; ASHURST, H. 1984. The effects of nuptiality, contraception and breastfeeding on fertility in developing countries. International Family Planning Perspectives 10(3): 8692

COALE, A.J. AND TRUSSELL, T.J . 1974. Model fertility schedules: variations in the age structure of childbearing in human populations. Population Index 40: 195-258.

CROGNIER, E. 1996. Behavioral and environmental determinants of reproductive success in traditional Moroccan Berber groups. American J ournal of Physical Anthropology 100(2): 181-90.

CROGNIER, E.; ELIZONDO, S.; CARATINI, A.L.; ZUBIETA, N.I.; NIBORSKY, R.; CARNESE, R.F. 1996. Los comportamientos reproductivos de la población 
mapuche de la provincia de Río Negro. Revista Argentina de Antropología Biológica 1(1): 86-97.

CROGNIER, E.; BAALI, A.; HILALI, M.K. 2001. Do "helpers at the nest" increase their parents' reproductive success? American J ournal of Human Biology 13(3): 365-73.

CROSS, H.E. AND MCKUSICK, V.A. 1970. Amish demography. Social Biology 17: 83-101.

EATON, J.W. AND MAYER, A.J . 1953. The social biology of very high fertility among the Hutterites. The demography of a unique population. Human Biology 25: 206-64.

FELT, J.C.; RIDLEY, J.C.; ALLEN, G.; REDEKOP, C. 1990. $\mathrm{High}$ fertility of old colony Mennonites in Mexico. Human Biology 62(5): 689-700.

FORT, A.L. 1989. Investigating the social context of fertility and family planning: a qualitative study in Peru. International Family Planning Perspectives 15(3): 8895.

GARCÍA-MORO, C.E.; TOJA, D.I.; WALKER, P.L. 1997. Marriage patterns of California's early SpanishMexican colonists (1742-1876). J ournal of Biosocial Science 29(2): 205-218.

GREKSA, L.P. 2002. Population growth and fertility patterns in an Old Order Amish settlement. Annals of Human Biology 29(2): 192-201.

HENRY, L. 1961. Some data on natural fertility. Eugenics Quaterly 8: 81-91.

HERNÁNDEZ, M.; GARCÍA-MORO, C.; MARTINIC, M. 1998. Evolución demográfica de la población de Tierra del Fuego (Región de Magallanes). Anales del Instituto de la Patagonia, Serie Ciencias Humanas, 26: 41-68.

HEWNER, S.J . 1998. Fertility, migration and mortality in an old order Amish community. American J ournal of Human Biology 10: 619-628.

JONES, E.F. 1971. Fertility decline in Australia and New Zealand, 1861-1936. Population Index 301.

KNODEL, J.; WILSON, C. 1981. The secular increase in fecundity in German village populations: an analysis of reproductive histories of couples married (17501899). Population Studies 35: 53-84.

LI, C.C. 1975. Path analysis. A primer. The Boxwood Press. Pacific Grove, California.

LOEBNER, H. AND DRIVER, E.D. 1973. Differential fertility in Central India: a path analysis. American J ournal of Physical Anthropology 10(3): 329-50.

LUNA, F. 1981. Biología de la población alpujarreña: evolución y estructura. Universitat de Barcelona.

LUNA, F. AND FUSTER, V. 1990. Reproductive pattern in a rural Mediterranean population: La Alpujarra, Spain. J ournal of Biosocial Science 22: 501-6.

MARTINIC, M. 1982. La Tierra de los Fuegos. Municipalidad de Porvenir. Porvenir.

MARTINIC, M. 1992. La inmigración croata en Magallanes. A pellidos y origen regional. Anales del Instituto de la Patagonia, Serie Ciencias Humanas, 21: 17-32.

MARTINIC, M. 1999. La inmigración chilota en Magallanes. Apreciación histórica sobre sus causas, características y consecuencias. Anales del Instituto de la Patagonia, Serie Ciencias Humanas, 27:27-47.

MINEAU, G.P.; BEAN, L.L.; SKOLNICK,M.P. 1979. Mormon demographic history II: The family life cycle and natural fertility. Population Studies 33(3): 429-446.

NONAKA, K.; MIURA, T.; PETER, K. 1994. Recent fertility decline in Dariusleut Hutterites: an extension of Eaton and Mayer's Hutterite fertility study. H uman Biology 66(3): 411-20.

NORTH, K.E.; MACCLUER, J.W.; COWAN, L.D.; HOWARD, B.V. 2000. Gravidity and parity in postmenopausal American Indian women: The Strong Heart Study. Human Biology 72(3): 397-414.

PASCUAL, J. 2004. Caracterización de la historia reproductora y análisis de la fecundidad de las mujeres de Tierra del Fuego. Tesis doctoral. Universidad de Barcelona MS.

PASCUAL, J .; GARCÍA-MORO, C.; HERNÁNDEZ, M. 2005. Biological and behavioral determinants of fertility in Tierra del Fuego. American J ournal of Physical Anthropology, 127(1): 105-113.

SMITH, R.M. 1988. Natural fertility in Pre-industrial Europe. Natural human fertility. Social and biological determinants 70-88. Diggory, P. etal.(eds). MacMillan Press and The Eugenics Society. London.

WOOD, J.W. 1994. Dynamics of human reproduction. Biology, biometry, demography. Aldine de Gruyter. New York.

ZODGEKAR, A.V. 1980. The fertility transition in the nonMaori population of New Zealand. Journal of Biosocial Science 12(2): 165-78. 